\title{
Cost allocation model for distribution networks considering high penetration of distributed energy resources
}

\author{
Tiago Soares, Fábio Pereira, Hugo Morais, Zita Vale
}

\begin{abstract}
A B S T R A C T
The high penetration of distributed energy resources (DER) in distribution networks and the competitive environment of electricity markets impose the use of new approaches in several domains. The network cost allocation, traditionally used in transmission networks, should be adapted and used in the distribution networks considering the specifications of the connected resources. The main goal is to develop a fairer methodology trying to distribute the distribution network use costs to all players which are using the network in each period. In this paper, a model considering different type of costs (fixed, losses, and congestion costs) is proposed comprising the use of a large set of DER, namely distributed generation (DG), demand response (DR) of direct load control type, energy storage systems (ESS), and electric vehicles with capability of discharging energy to the network, which is known as vehicle-to-grid (V2G). The proposed model includes three distinct phases of operation. The first phase of the model consists in an economic dispatch based on an AC optimal power flow (AC-OPF); in the second phase Kirschen's and Bialek's tracing algorithms are used and compared to evaluate the impact of each resource in the network. Finally, the MW-mile method is used in the third phase of the proposed model. A distribution network of 33 buses with large penetration of DER is used to illustrate the application of the proposed model.
\end{abstract}

Keywords:

Bialek's tracing method

Distributed energy resources

Kirschen's tracing method

MW-mile

Network tariffs allocation

Topological distribution factors

\section{Introduction}

\subsection{Background, methodology and aim}

The design and development of cost allocation methods applied to users of transmission systems is a topic widely studied, resulting in several different methods for cost allocation. However, at the distribution system level, the cost allocation topic has been the target of deep study because its technical and operation characteristics are different from transmission systems requiring the development of new methodologies.

Traditionally, the operation costs in distribution systems are allocated to consumers connected in the network based on average operation costs [1]. With the increasing penetration of distributed energy resources (DER) in distribution systems, the traditional cost allocation methods are no longer valid, due to different directions of power flow in distribution systems caused by DER [2]. Thus it is necessary to develop new methodologies more adequated for the new operation paradigm.

In fact, the actual power systems are no more characterized by a central generation units connected to transmission systems and a passive consumers most of them connected to medium and low voltage distribution networks. This operation paradigm has gradually changed to a more decentralized one. Nowadays, most of the power systems are characterized by the high penetration of distributed generation connected in all voltage levels, the existence of storage systems (pumped hydro power plants and few batteries based systems), the growing introduction of electric vehicles and the active participation of consumers through the demand response programs and a more conscience concerning the efficiency use of the energy. Taking this reality into account, the methods traditionally used to determine the costs allocation of the distribution 


\section{Nomenclature}

\section{Parameters}

$\eta_{c} \quad$ grid-to-storage/vehicle efficiency

$\eta_{d} \quad$ storage/vehicle-to-grid efficiency

$B \quad$ imaginary part in admittance matrix (S)

$C \quad$ resource cost in period $t$ [m.u./kWh]

DF pay factor used to determine the fixed costs allocation for each type of resource

DN pay factor used to determine the network use costs allocation for each type of resource

$D L \quad$ pay factor used to determine the system losses costs allocation for each type of resource

$E \quad$ stored energy in the battery of storage system or vehicle at the end of period $t(\mathrm{kWh})$

$E_{\text {Initial }}$ energy stored in the battery of storage system or vehicle at the beginning of period $1(\mathrm{kWh})$

$E_{\text {Trip }} \quad$ energy consumption in the battery during a trip that occurs in period $t(\mathrm{kWh})$

$F \quad$ branch power flow $(\mathrm{kW})$

$G \quad$ real part in admittance matrix (S)

$L \quad$ branch Losses (kW)

LMP locational marginal price (m.u/kWh)

$N \quad$ number of resources

$S \quad$ apparent power flow in branch (kVA)

$T \quad$ total number of periods

$X \quad$ payment factor

$\bar{U} \quad$ voltage in polar form (V)

$\bar{y} \quad$ series admittance of line that connects two buses (S)

$\overline{y_{s h}} \quad$ shunt admittance of line that connects two buses (S)

\section{Superscript}

$\begin{array}{ll}\text { Fixed } & \text { fixed costs (m.u.) } \\ \text { Loss } & \text { losses costs (m.u.) } \\ \text { NetUse } & \text { network use costs (m.u.) }\end{array}$

Indices

$i, j \quad$ node index

$d g \quad$ distribution generation index

sp external suppliers index

st energy storage system index

$t$ time index in hours (h)

$v 2 g \quad$ vehicle-to-grid index

\section{Variables}

$\theta \quad$ voltage angle

C cost (m.u.)

$P \quad$ active power $(\mathrm{kW})$

$Q \quad$ reactive power $(\mathrm{kVAr})$

TC total allocation cost (m.u.)

$V \quad$ voltage magnitude (V)

Y binary variable

\section{Subscript}

inefficiency costs
fixed component of cost function (m.u./h)

$B$ linear component of cost function (m.u./kWh)

Branch branch

C quadratic component of cost function (m.u. $/ \mathrm{kWh}^{2}$ )

$\mathrm{Ch}$ storage or V2G charge process

Dch storage or V2G discharge process

$D G \quad$ distribution generation

$D R A \quad$ active power reduction of load

$D R \_B \quad$ active power curtailment of load

$\begin{array}{ll}\text { GCP } & \text { generation curtailment power } \\ \text { Load } & \text { loads } \\ \text { LTC } & \text { loads total cost } \\ \text { Max } & \text { upper bound limit } \\ \text { Min } & \text { lower bound limit } \\ \text { NSD } & \text { non-supplied demand } \\ \text { SP } & \text { external supplier } \\ \text { V2G } & \text { vehicle-to-grid }\end{array}$

network, in which the consumers pay all the costs, are no more adequate. A new methodology is proposed in this paper for the costs allocation in distribution network, taking into account the new operation paradigm with large penetration of several types of DER. The main goal of the proposed method is to distribute the costs fairer to all players connected to the distribution networks taking into account the effective use of the network in each period (15, 30 or $60 \mathrm{~min})$. As mentioned, the proposed methodology considers several types of DER, namely distributed generation (DG); direct load control demand response (DR); energy storage systems (ESS); and electric vehicles with the capability to charge and discharge energy, usually referred as vehicle-to-grid (V2G) resources.

The methodology considers the combination of three different cost allocation methods with the aim of take advantage of the main qualities of each one, to develop a more fairly cost allocation model. The methodology comprises three levels. The first level consists in the energy resources schedule optimization considering the merit order, in this case the operation cost. By considering an AC OPF it is also possible to obtain the locational marginal prices (LMP) in each bus, including the energy LMP, the losses LMP, and the congestion LMP (marginal method). The second level intends to determine the share/impact that each energy resource has on the network power flow (tracing method). Two different approaches based on the proportional sharing principle are tested and compared to determine the impact of each resource in the network. The third level consists in the application of allocation costs method to each type of resource (variant of MW-mile method).

\subsection{Literature review and specific contributions}

The cost allocation is a topic widely studied in transmission networks [3-24]. However, the increasing penetration of DER at distribution level forces the need to adapt traditional cost allocation methods used in transmission system to the distribution level. In general, the cost allocation methods for transmission systems may be classified into three distinct categories: nodal marginal methods [3-7]; rolled-in methods [8-11]; embeddedmethods [12-24]. The cost allocation based on nodal marginal pricing for transmission systems is presented in [3-5], in which are considered the long-term and short-term marginal costs related to energy, reliability, investments and demand side. Similar approaches for distribution networks considering distributed generation are proposed in [6,7]. These approaches have some limitations. In [6] the tariff scheme only considers the consumers disregarding the generation units. In [7] the fixed costs scheme for demand and DG resources are considered but only for extreme scenarios.

The rolled-in methods are characterized by their easy implementation ensuring return on the total system operation costs. These methods allow getting a tariff based on the average cost of the system. The postage stamp [8,9], contract path [10], and 
mean participation factors [11] are the most important rolled-in methods.

The embedded methods based on power flow analysis are characterized by the inclusion of network technical characteristics, resulting in a proper cost allocation method to each entity involved in the system. In this class, there are several cost allocation methods. The MW-mile [12] method is widely used in transmission networks, in which considers the changes in MW transmission flow and transmission line length in miles. Several other methods were developed based on MW-mile method, such as the unused capacity [13], the zero counter-flow [14], the dominant flow [15], and the MVA-mile [16]. Based on the power flow, the equivalent bilateral exchange method for cost allocation is proposed in [17]. In this method, it is considered that a portion of each generator, being the portion divided uniformly by all generators, supplies each load. Thus, each generator provides a portion of each load uniformly divided by all the loads of the system. The general agreement on parallel paths method [18] consists in a set of rules to reward the system operator considering the impact of each resource in the network. This method is based on getting parallel paths of the power flow when a single contract path between two parts is not good enough. Thus, the method considers an initial set of criteria and rules based on system reliability to perform parallel paths that are used to remunerate the system. The rated system path [19] is a method based on the study of the transmission capacity in the system. The transmission capacity is obtained by conducting several studies of the power flows, considering different situations of system operation which results in the cost allocation of the system. The Zbus method is based on circuit theory and it can determine how an injection of power of a given resource uses the network. Thus, it is possible to allocate the costs of network usage to the energy resources [20]. Others embedded cost allocation methods such as the generalized distribution factors [15], Bialek's [21], and Kirschen's [22] tracing algorithms are based on the definition of distribution factors in order to know the share that each user has in the network. An hybrid method called "Amp-mile" [23] combines the use of power flow distribution factors in order to know the impact that each user has on each network branch, with some characteristics of the MW-mile. A complete distinct approach based on game theory is proposed in [24], which allocates the cost of DG embedded distribution network based on Nucleolus and Shapley value approaches. For distribution systems a methodology considering Bialek's tracing algorithm to trace the power flow and a variant of MW-mile to tariff each resource use on the network is proposed by [25].

As it is possible to see by the large number of different techniques proposed in literature, the network costs allocation is a very important topic for power systems. However, most of the techniques were developed to be used at the transmission level considering a centralized generation approach and passive consumer's behavior. These traditional characteristics will change in future power systems. Furthermore, most of the techniques use different variants of MW-mile to tariff each resource without considering improvements on the efficiency of the method. The original MW-mile model presents some inefficient results as identified in [15]. The MW-mile inefficiency means that some costs are not allocated to any player. To avoid this inefficiency it is necessary develop some inefficiency compensation scheme improving the sustainability of the model as well as properly and fairly allocate the costs to all resources of the system. Moreover, future distribution systems will include several types of different generation and consumers resources, such as V2G resources. V2G resources increase the complexity of the distribution system management. The ability to be a consumer or a generation resource should be taken into account in order to properly allocate impact costs of this resource in the system. The main objective of the present work is to propose a model able to properly and fairly allocate the distribution network operation costs to all players connected in this network. The main contributions of this paper are:

(a) To propose a model to tariff distribution network use, considering large penetration of distributed energy resources, namely distributed generation, demand response, energy storage systems, and vehicle-to-grid.

(b) To propose a model to cost allocation of storage base distributed resources, such as ESS and V2G, considering the ability act as a generator or as a consumer among time horizon.

(c) To design a complete model able to take advantage of three different known cost allocation methods. Marginal method to obtain economic signals for network use and losses costs, tracing method to proportional determine the share for each type of resource, and a variant of MW-mile method to allocate costs to resources considering the marginal and tracing methods.

(d) To implement, evaluate and compare two different methods of tracing power flow (Kirschen's and Bialek's tracing algorithms) considering future distribution network characteristics.

(e) To integrate a new inefficiency penalty scheme to improve MWmile method and the full sustainability of the model avoiding the existence of costs not allocated to any player.

\subsection{Paper organization}

The paper is structured as follows: Section 2 presents the detailed tariff model definition considering all the assumptions and features of the model; Section 3 illustrates the model operation in a distribution network considering the large penetration of DER; Section 4 exposes the most important conclusions.

\section{Distribution network tariffs definition}

The proposed methodology consists in three main steps represented in Fig. 1. The method starts by obtaining the locational marginal prices (LMP) [26-28] through an economic dispatch algorithm. Then is determined the network use by each energy resource based on distribution coefficients models. The last stage deals with the network allocation costs for each resource based on a variant of the MW-mile method. The combination of the advantages of three different methods leads to a more efficient, properly and fairly model to allocate distinct distribution network costs to all type of distributed energy resources.

\subsection{First step - energy resources scheduling and locational marginal prices definition}

To obtain locational marginal prices in each bus an economic dispatch algorithm is used to schedule the DER units connected to the distribution network, based on their operation cost and on the contracts established with these DER. The objective function has the main goal of minimizing operation costs of distribution system operator taking into account several types of DER. The DER considered in the present methodology are the distributed generation (DG), the active participation of consumers in direct load control demand response events (DR), electric vehicles with capacity to charge and discharge energy (V2G), and energy storage systems (ESS). Additionally, part of the power demand is supplied by external entities through the transmission network. These external entities are called external suppliers in this method. The external suppliers, represents the suppliers connected to upstream network levels of the distribution network that supply energy to satisfy the demand required in the distribution network. This energy can be bought in different markets sessions or in bilateral contracts. In 


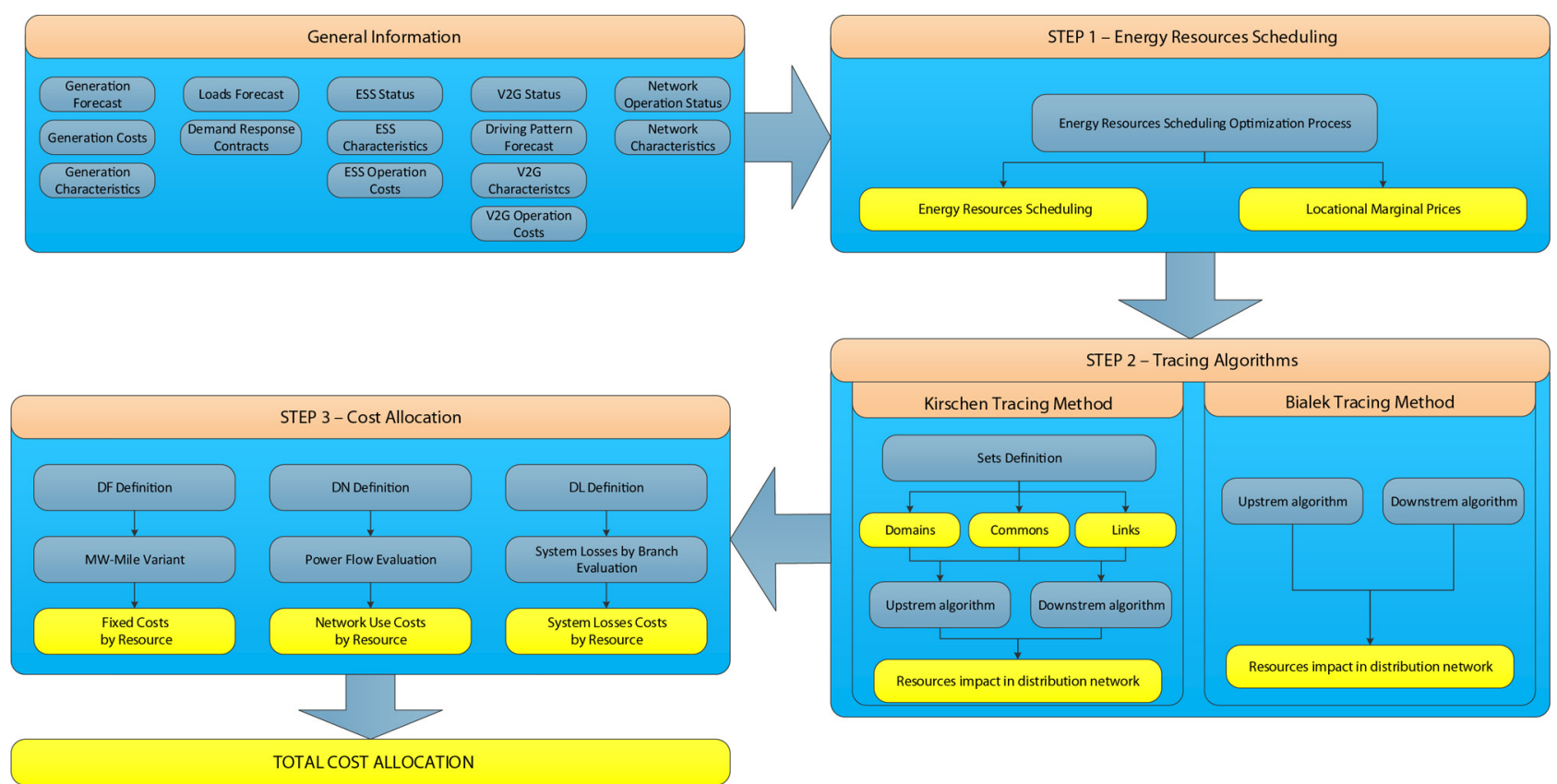

Fig. 1. Diagram of the proposed model.

a normal operation (without congestion situations and when the consumption is higher than the generation), the price of energy supplied by the external suppliers will impose the energy locational marginal price. However, some DER units have "feed-in" contracts imposing the delivery of all generated energy. In specific situations, such as when internal resources with "feed-in" contracts produce more energy than required, the energy LMP will be imposed by the price of these resources and not by the external suppliers. The energy resources scheduling can be solved as an optimization problem considering the minimization of the operation costs (1). of each period in each V2G. To determine the amount of energy, it is usual to consider typical daily travel profile of each V2G as well as efficiency of charge and discharge energy in the grid. The energy stored on the battery of each V2G requires minimum (16) and maximum (17) limits. Charge (18) and discharge (19) rates present itself maximum limit. Charge and discharge energy for each V2G cannot occur at same time, so non-simultaneity of charge and discharge (20) is ensured. Technical constraints for energy storage systems (20)-(26) follow the same principle of constraints regarding V2G resources. The main difference between both resources is

$$
\begin{aligned}
& \text { Minimize } f=\min \\
& \sum_{t=1}^{T}\left[\sum_{d g=1}^{N_{D G}}\left(\begin{array}{l}
B_{D G(d g, t)} \times c_{A(d g, t)}+P_{D G(d g, t)} \times c_{B(d g, t)}+ \\
P_{D G(d g, t)}^{2} \times c_{C(d g, t)}+P_{G C P(d g, t)} \times c_{G C P(d g, t)}
\end{array}\right)+\sum_{v 2 g=1}^{N_{V 2 G}}\left(\begin{array}{l}
P_{D c h(v 2 g, t)} \times c_{D c h(v 2 g, t)} \\
-P_{C h(v 2 g, t)} \times c_{C h(v 2 g, t)}
\end{array}\right)\right. \\
& \left.+\sum_{l=1}^{N_{L}}\left(\begin{array}{c}
P_{D R \_A(l, t)} \times c_{D R \_A(l, t)}+P_{D R \_B(l, t)} \times c_{D R \_B(l, t)} \\
+P_{N S D(l, t)} \times c_{N S D(l, t)}
\end{array}\right)+\sum_{s t=1}^{N_{S T}}\left(\begin{array}{c}
P_{D c h(s t, t)} \times c_{D c h(s t, t)} \\
-P_{C h(s t, t)} \times c_{C h(s t, t)}
\end{array}\right)+\sum_{s p=1}^{N_{S P}} P_{S P(s p, t)} \times c_{S P(s p, t)}\right]
\end{aligned}
$$

The optimal energy resources scheduling includes several constraints related with the DER units and the distribution network [29]. An AC-OPF [30] is included to determine the active and reactive power that flows in each branch of the distribution system. Active balance (2) is determined based on all resources available in the system. Reactive balance (3) is determined considering only the resources able to provide reactive power as distributed generation and external suppliers. The AC-OPF determines the bus voltage magnitude (4) and voltage angles (5) taking into account the branch thermal limits (6) and (7). Besides AC-OPF constraints it is also considered technical constraints regarding the intrinsic characteristics of each type of resource (8)-(26). For external suppliers active (8) and reactive (9) limits of power delivery is considered. Distribution generators comprise active generation limits (10) and generation curtailment in active power (11), as well as reactive power (12). Demand reduction (13) and curtailment (14) through direct load control of demand response. Vehicle-to-grid resources will be an important resource in future distribution systems, but it increases the complexity of the problem (15)-(20). In this way, is essential to optimize the amount of energy stored (15) at the end that energy stored systems does not need reserve energy for travel, since they are not vehicles.

\subsection{Second step - tracing algorithms}

Based on the resources scheduling results and on the obtained LMPs, the second step of the proposed methodology aims to determine the contribution of each resource in the use of each network branch. In order to determine the resource contribution, two different techniques were implemented and tested. The first one is the Kirschen's tracing method proposed in [22]. This technique defines the assumptions of domains, commons and links in order to determine the contribution of each resource in the network power flow. The second technique, called Bialek's tracing method uses the topological distribution factors, which consider the proportional sharing of a network node assumption to determine the contribution of each resource in the network power flow [21]. Both techniques consider the proportional sharing principle. 
Furthermore, the Bialek's tracing method uses two distinct algorithms (upstream and downstream-looking algorithms) in order to determine the impact of each generation and consumption resource in the network power flow.

Using these techniques to determine the impact that each resource has in each network branch is essential for the proper functioning of the proposed model. The model includes the use of these techniques in all types of DER in order to account the impact of such resources in a distribution system. DG, DR, ESS and V2G resources are considered in this model. Additionally, the loads are also considered as an active player in the cost allocation model. This means that in the proposed method the loads can be seen as an energy resource. Regarding the demand response programs, this resource is seen as a generation resource in the scheduling process, being considering the contracts costs. However, regarding the network use, the DR represents a load reduction. By taking this aspect into account, the DR is not included directly in the cost allocation problem, yet it is deducted from the load consumption. In practice, this means a negative cost due to the less load contribution in the cost distribution. In fact, the use of DR will improve the global system efficiency, at least during the DR use periods. The power flow caused by the load entity considers the difference between the initial energy required by the consumer, minus the power curtailed due to the participation on DR programs.

\subsection{Kirschen's tracing method}

The Kirschen's tracing method is a technique which aims to determine the impact that the generation and consumption resources have on the use of the distribution network. This technique is based on a set of definitions [22]:

- Domains - set of buses that are reached by power produced by particular generator.

- Commons - set of contiguous buses supplied by the same set of generators.

- Links - branches that connect with the commons.

This set of assumptions results in a simplification of the graphical structure of the network. Thus, it is used the proportional sharing principle to determine the share of each resource in each common, link, loads, and in the power flow of each common. This simplification may lead to imperfect results, especially when the network has a meshed structure and/or there is opposite power flow in the direction of the main flow of the system. Distribution networks are typically operated in radial mode and with unidirectional power flow. However, with high penetration of DER, the opposite power flows can occur in several periods throughout the day.

The method can be applied to all kinds of resources. However, there are two different algorithms (upstream and downstreamlooking algorithms) that are used to trace the power for generation and consumption resources. The upstream-looking algorithm determines the share of generation resources, while the downstream-looking algorithm determines the impact of the consumption resources in the system.

\subsection{Bialek's tracing algorithm}

The Bialek's approach consists in the use of topological distribution factors in order to determine the share of the energy resources in the power system [21]. This method is based on the proportional share principle of power that considers the incoming flows and outflows in a node. This approach assumes that all topological distribution factors are positive, so the model is immune to counterflow problems in the branches that may occur in networks with high DER penetration. Similar to the Kirschen's method, this technique also uses two tracing flow algorithms, upstream-looking algorithm to determine the share of generation resources, and downstream-looking algorithm to determine the share of consumption resources.

\subsection{Third step - cost allocation}

The cost allocation model corresponds to the last stage of the proposed methodology that is based on the MW-mile approach $[12,15]$. Indeed, this stage uses a variant of the MW-mile method proposed in [15] in order to allocate the system costs. The proposed MW-mile variant tends to be fairer to the resources than the traditional MW-mile approach. The proposed variant of MWmile allocates the costs to the resource according to its impact in each network branch, while the traditional method uses the branch length or the total capacity of the branch. In addition, the proposed variant of MW-mile takes into account the previous stages of the model, where marginal and tracing methodologies are applied. Thus, the proposed cost allocation model for each resource comprises a combination of three different methods studied in literature.

This stage considers the allocation of three distinct kinds of costs for each resource based on the share of each resource in the system. The costs are divided into fixed, network use and losses costs. The total cost comprises the sum of the fixed, network use, and losses costs for each resource. The sum of each total cost results in the global allocation costs associated with the system.

$$
\begin{aligned}
& T C_{(d g)}=\sum_{i=1}^{N_{B}} \sum_{i=j}^{N_{B}}\left(C_{(i, j, d g)}^{\text {Fixed }}+C_{(i, j, d g)}^{\text {NetUse }}+C_{(i, j, d g)}^{\text {Loss }}\right) \\
& T C_{(l)}=\sum_{i=1}^{N_{B}} \sum_{i=j}^{N_{B}}\left(C_{L T C(i, j, l)}^{\text {Fixed }}+C_{L T C(i, j, l)}^{\text {NetUse }}+C_{L T C(i, j, l)}^{\text {Loss }}\right) \\
& T C_{(v 2 g)}=\sum_{i=1}^{N_{B}} \sum_{i=j}^{N_{B}}\left(C_{(i, j, v 2 g)}^{\text {Fixed }}+C_{(i, j, v 2 g)}^{\text {NetUse }}+C_{(i, j, v 2 g)}^{\text {Loss }}\right) \\
& T C_{(s t)}=\sum_{i=1}^{N_{B}} \sum_{i=j}^{N_{B}}\left(C_{(i, j, s t)}^{\text {Fixed }}+C_{(i, j, s t)}^{\text {NetUse }}+C_{(i, j, s t)}^{\text {Loss }}\right)
\end{aligned}
$$

\subsection{Fixed costs}

The fixed costs are related to the network operation and maintenance costs, as well as to the network initial investment costs. Contribution of DG in fixed costs of each branch is determined by knowing the power flow in branch, the contribution for DG in power flow of the branch, fixed cost of the branch and payment factor for DG units. The pay factor $D F_{(i, j, d g)}$ is used to establish the contribution that each kind of resource has in fixed costs. The factor ranges from 0 to 1 and it is imposed by the distributed network operator based on strategic, political and environmental reasons. Similarly, the fixed costs for loads are allocated based on the same principle. The demand response programs will decrease the load consumption. Consequently, the payment fees applied to the loads will be reduced. This means an indirect incentive to increase consumers participation on these programs.

$C_{(i, j, d g)}^{\text {Fixed }}=\frac{D F_{(i, j, d g)} \times C_{\text {Branch }(i, j)}^{\text {Fixed }}}{F_{(i, j)}} \times X_{D G}$

Regarding the ESS and V2G resources, the principle to determine the fixed costs is similar to the DG resources. However, it is necessary to consider the charge and discharge capability of these resources. Therefore, the costs for charge and discharge must be 
considered, based on the participation that charge and discharge ability has on network power flow.

$$
\begin{aligned}
C_{(i, j, v 2 g)}^{\text {Fixed }}= & \left(\frac{D F_{C h(i, j, v 2 g)} \times C_{\text {Branch }(i, j)}^{\text {Fixed }}}{F_{(i, j)}}+\frac{D F_{D c h(i, j, v 2 g)} \times C_{\text {Branch }(i, j)}^{\text {Fixed }}}{F_{(i, j)}}\right) \\
& \times X_{V 2 G}
\end{aligned}
$$

The distribution of the payment factor for different kind of resources can lead to inefficiency in the total cost allocation of the system. The difference between the fixed costs of the branch and the sum of all costs determined for each resource will give the economic inefficiency of the model for that branch.

$$
\begin{aligned}
& C_{\psi(i, j)}^{\text {Fixed }}=C_{\text {Branch }(i, j)}^{\text {Fixed }} \\
& -\left[\sum_{d g=1}^{N_{D G}} C_{(i, j, d g)}^{\text {Fixed }}+\sum_{l=1}^{N_{L}} C_{(i, j, l)}^{\text {Fixed }}+\sum_{s t=1}^{N_{S T}} C_{(i, j, s t)}^{\text {Fixed }}+\sum_{v 2 g=1}^{N_{V 2 G}} C_{(i, j, v 2 g)}^{\text {Fixed }}\right]
\end{aligned}
$$

This implies that the system operator cannot be fully refunded for the use of the system by the energy resources. In order to address the inefficiency in the distribution of the payment factors, it is assumed that the costs associated with the inefficiency will be supported by the loads entities. This means, an extra incentive to increase the consumption efficiency.

$C_{L T C(i, j, l)}^{\text {Fixed }}=\frac{D F_{(i, j, l)}}{\sum_{l=1}^{N_{L}} D F_{(i, j, l)}} \times C_{\psi(i, j)}^{\text {Fixed }}+C_{(i, j, l)}^{\text {Fixed }}$

\subsection{Network use costs}

The network use costs are associated, as the name suggests, with the use of network related to the power flow in each branch of the network, i.e., the costs are determined taking into account the impact of the power flow in each network branch. This cost is distributed among all resources (DG, ESS, V2G and loads) considering the impact of each one in the network. The network use cost by branch for distribution generation is determined based on DG payment factor with power flow on branch and total the cost of network use of the branch, while it is considered the distribution factor for $D G D N_{(i, j, d g)}$. $D N$ factor is similar to the $D F$ factor used to determine the fixed cost. The same value can be used for both.

$C_{(i, j, d g)}^{\text {NetUse }}=\frac{D N_{(i, j, d g)} \times C_{B r a n c h(i, j)}^{\text {NetUse }}}{F_{(i, j)}} \times X_{D G}$

As for the fixed costs, for electric vehicles and storage systems, it is important to consider both the charge and discharge processes. Thus, it is considered charge and discharge distribution factors to determine the contribution of the electric vehicles in the network use costs. The same principle is applied for storage systems.

$$
\begin{aligned}
C_{(i, j, v 2 g)}^{\text {NetUse }}= & {\left[\frac{D N_{C h(i, j, v 2 g)} \times C_{B r a n c h}^{\text {Neti } i, j)}}{F_{(i, j)}} \times \frac{D N_{D c h(i, j, v 2 g)} \times C_{\text {Branch }(i, j)}^{\text {NetUse }}}{F l(i, j)}\right] } \\
& \times X_{V 2 G}
\end{aligned}
$$

The cost $C_{B r a n c h(i, j)}^{\text {NetUse }}$ can be obtained considering the difference between the LMPs in the bus connected by the branch. In usual operation, the difference between LMP of each bus will reflect the LMP regarding the losses. However, in critical operation conditions with situations of congestion, the LMP's difference will also reflect the congestion LMP. In order to penalize the use of network near by the boundaries, $C_{B r a n c h(i, j)}^{\text {NetUse }}$ can be determined as

$$
\left\{\begin{array}{l}
C_{B \operatorname{Rranch}(i, j)}^{\text {NetUse }}=\left|L M P_{(j)}-L M P_{(i)}\right| ; \quad \text { if } \frac{F_{(i, j)}}{F_{\operatorname{Max}(i, j)}}(\%) \leq 85 \% \\
C_{\text {Branch }(i, j)}^{\text {NetUse }}=5 \times\left|L M P_{(j)}-L M P_{(i)}\right| ; \quad \text { if } 85 \%<\frac{F_{(i, j)}}{F_{\operatorname{Max}(i, j)}}(\%) \leq 98 \% \\
C_{\text {Branch }(i, j)}^{\text {NetUse }}=10 \times\left|L M P_{(j)}-L M P_{(i)}\right| ; \quad \text { if } \frac{F_{(i, j)}}{F_{\operatorname{Max}(i, j)}}(\%)>98 \%
\end{array}\right.
$$

The main idea it is to penalize the use of the network near the boundaries. If the use of the network was higher than $85 \%$ of its capacity $a S_{\operatorname{Max}(i, j)}$, the cost will be five times higher, if the use of the network was higher than $98 \%$, the network use cost increases ten times. Thus, the resources are actively encouraged to contribute to system's efficiency.

As fixed costs, the method can lead to inefficiencies due to the mathematical rationality between distribution factor and payment factor. Thus, the costs of system inefficiency related to power flow costs can be determined as

$$
\begin{aligned}
& C_{\psi(i, j)}^{\text {NetUse }}=C_{\text {Branch }(i, j)}^{\text {NetUse }} \\
& -\left[\sum_{d g=1}^{N_{D G}} C_{(i, j, d g)}^{\text {NetUse }}+\sum_{l=1}^{N_{L}} C_{(i, j, l)}^{\text {NetUse }}+\sum_{s t=1}^{N_{S T}} C_{(i, j, s t)}^{\text {NetUse }}+\sum_{v 2 g=1}^{N_{V 2 G}} C_{(i, j, v 2 g)}^{\text {NetUse }}\right]
\end{aligned}
$$

where the difference of the branch cost for network use and all the costs for network use by each type of resource results in inefficiency cost to be charged in order to maintain economic balance of system operator.

Moreover, the costs of system inefficiency related to the network use costs are allocated to the loads. Thus, total power flow costs for loads are determined taking into account the addition of inefficiency costs to the already cost for the use of the network.

$C_{L T C(i, j, l)}^{P F}=\frac{D F_{(i, j, l)}}{\sum_{l=1}^{N_{L} D F_{(i, j, l)}}} \times\left(C_{\psi(i, j)}^{\text {NetUse }}\right)+\left(C_{(i, j, l)}^{\text {NetUse }}\right)$

\subsection{Losses costs}

The system losses cost is allocated to each resource according to the impact that each one has on losses. The proposed methodology determines the share that each resource has in the branch losses by rating it according to the losses cost $C_{\text {Branch }}^{\text {Loss } i, j)}$ of each branch. The system losses cost in a branch is determined by multiplying the power losses in that line, obtained at step 1, by the higher LMP value in the buses connected to the branch. The contribution of distribution generators to the system losses is determined as

$C_{(i, j, d g)}^{\text {Loss }}=\frac{D L_{(i, j, d g)} \times C_{B r a n c h(i, j)}^{\text {Loss }}}{L_{(i, j)}} \times X_{D G}$

where $D L_{(i, j, d g)}$ is the distribution factor of distribution generation considering losses flow in branch $i, j$, i.e., the contribution of DG unit to the losses in branch $i, j$. For loads impact determination, similar assumptions are made. For electric vehicles and storage systems determination of losses costs are determined as

$$
\begin{aligned}
C_{(i, j, v 2 g)}^{\text {Loss }}= & {\left[\frac{D L_{C h(i, j, v 2 g)} \times C_{\text {Branch }(i, j)}^{\text {Loss }}}{L_{(i, j)}} \times \frac{D L_{D c h(i, j, v 2 g)} \times C_{\text {Branch }(i, j)}^{\text {Loss }}}{L_{(i, j)}}\right] } \\
& \times X_{V 2 G}
\end{aligned}
$$

where charge and discharge distribution factor for losses in each branch it is considered. 


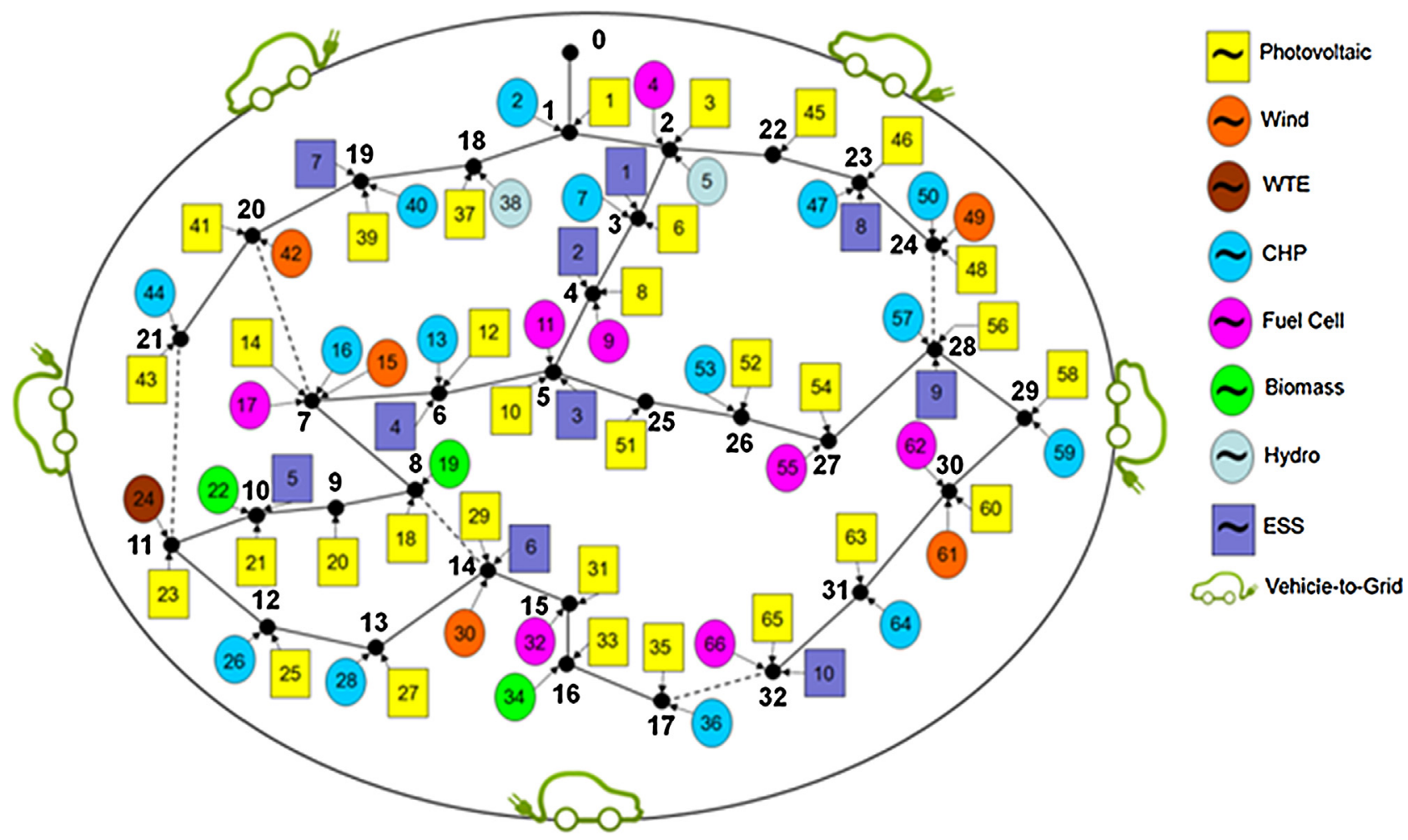

Fig. 2. Network configuration adapted from [31].

The losses costs related to the system inefficiency are given

$$
\begin{aligned}
& C_{\psi(i, j)}^{\text {Loss }}=C_{B r a n c h(i, j)}^{\text {Loss }} \\
& -\left[\sum_{d g=1}^{N_{D G}} C_{(i, j, d g)}^{\text {Loss }}+\sum_{l=1}^{N_{L}} C_{(i, j, l)}^{\text {Loss }}+\sum_{s t=1}^{N_{S T}} C_{(i, j, s t)}^{\text {Loss }}+\sum_{v 2 g=1}^{N_{V 2 G}} C_{(i, j, v 2 g)}^{\text {Loss }}\right]
\end{aligned}
$$

The inefficiency costs of the system are allocated to the load entity. In this way, the determination of the total losses cost for each load entity in the system is determined as

$C_{L T C(i, j, l)}^{\text {Loss }}=\frac{D F_{(i, j, l)}}{\sum_{l=1}^{N_{L} D F_{(i, j, l)}}} \times C_{\psi(i, j)}^{\text {Loss }}+C_{(i, j, l)}^{\text {Loss }}$

where total costs for load entities consider not only the impact of loads in system losses, but also inefficiency costs related to the system losses.

\section{Case study}

A case study considering the simulation of the proposed model based on a distribution system with large penetration of DER is described. The case study is divided into two sections - the case study characterization and the results analysis.

\subsection{Case characterization}

The case study is conducted based on a distribution network with 33 buses [31], taking into account a scenario of high penetration of distributed energy resources [32], as shown in Fig. 2. The tested network includes 66 DG units with different generation technologies, namely 32 photovoltaic systems, 15 combined heat and power (CHP), 8 fuel cell systems, 5 wind turbines, 3 biomass plants, 2 small hydro, and 1 waste-to-energy (WTE) units. The network is connected to the transmission system through the bus 0 . There are
32 consumption points throughout the network representing the medium/low voltage (MV/LV) power transformers. The consumers are aggregated at these consumption points. Similarly, demand response programs can be scheduled by consumption point and not directly by each consumer. Two types of loads are considered for DR participation, namely the continuous regulation loads, with capability of reducing the consumption, and the discrete loads (ON/OFF) that are used for load curtailment. The network contains 10 ESS and 50 V2G resources able to charge and discharge energy. The number of electric vehicles is relatively small, yet enough to evaluate the impact of this type of resource in the cost allocation methodology. The total generation capacity and the resources operation costs are presented in Table 1. In this table the ESS and V2G total storage capacity are also represented. The costs are applied in the discharge process and only consider the degradation costs of the batteries. The values are obtained considering the studies presented in $[33,34]$.

The system operation costs should be allocated to each kind of resource. Currently, most of the system operators allocate all the costs related to the network tariffs to consumers. However, some system operators (such as the Statnett SF in Norway or the NationalGrid in the UK) allocate more than thirty percent of the costs to generators and less than seventy percent to consumers [35]. In the proposed approach, the costs are allocated by generation and consumption equally. However, inefficiency penalties are allocated only to the consumers (40). Additionally, in some periods, besides the generation and the consumption resources, the scheduling of storage and electric vehicles charge and/or discharge can occur. To take care of these different operation scenarios, a variable costs share is used. Table 2 shows the share in four possible operation scenarios.

Regarding fixed costs $C_{\text {Branch }(i, j)}^{\text {Fixed }}$, there is no information on investment, operation, and maintenance costs for the considered network. In this way, and based on the Portuguese energy authority (ERSE) [36] a percentage value of the system operation cost is considered. In the present case study, the average daily operation 
Table 1

Resources characteristics.

\begin{tabular}{|c|c|c|c|c|c|c|}
\hline \multirow[t]{2}{*}{ Resources } & \multicolumn{3}{|c|}{ Installed/contracted capacity (kW) } & \multicolumn{3}{|c|}{ Resources operation cost (m.u./kWh) } \\
\hline & Minimum & Maximum & Total capacity & Minimum & Mean & Maximum \\
\hline Photovoltaic & 3 & 30 & 558 & 0.08000 & 0.13940 & 0.25400 \\
\hline Wind & 20 & 150 & 525 & 0.05000 & 0.06520 & 0.08000 \\
\hline $\mathrm{CHP}$ & 1 & 600 & 1200 & 0.00106 & 0.01790 & 0.06500 \\
\hline Biomass & 100 & 150 & 350 & 0.06000 & 0.26530 & 0.65000 \\
\hline WTE & 10 & 10 & 10 & 0.03000 & 0.04840 & 0.05600 \\
\hline Small hydro & 30 & 40 & 70 & 0.03200 & 0.04320 & 0.04900 \\
\hline Fuel cell & 10 & 50 & 235 & 0.09500 & 0.10210 & 0.11000 \\
\hline External supplier & - & - & 15,000 & 0.01500 & 0.06050 & 0.21000 \\
\hline Reduce & 7.15 & 250.19 & 834.83 & 0.10000 & 0.27270 & 0.80000 \\
\hline Cut & 7.15 & 147.50 & 633.89 & 0.09000 & 0.49920 & 1.20000 \\
\hline ESS capacity (MWh) & - & - & 1200 & 0.04500 & 0.05250 & 0.07000 \\
\hline V2G capacity (MWh) & - & - & 7828 & 0.04500 & 0.05250 & 0.07000 \\
\hline Load & 86.63 & 833.95 & 7245.20 & - & - & - \\
\hline
\end{tabular}

Table 2

Payment factor distribution.

\begin{tabular}{llrrr}
\hline Payment factor (\%) & DG and external suppliers & Load & Storage & V2G \\
\hline 1 & 50.0 & 50.0 & 0.0 & 0.0 \\
2 & 35.0 & 35.0 & 30.0 & 0.0 \\
3 & 35.0 & 35.0 & 0.0 & 30.0 \\
4 & 35.0 & 35.0 & 15.0 & 15.0 \\
\hline
\end{tabular}

cost is of 16,000 monetary units (m.u.), and fifteen percent is considered corresponding a daily fixed costs of 2400 m.u., and 100 m.u in each hour. These values can vary for different networks, and they can be higher in future smart grids due to the high investment in new control and protection equipment. However, the definition of these values is out of the scope of the present paper.

\subsection{Results}

The case study performs several results for each step of the model application. For first step it is performed an energy resource management of the distribution network with all resources included. The impact and contribution that each type of resource has on network power flow is shown in second step. Third step depicts all the costs allocated to each resource of the network, as well as the system remuneration for network use.

\subsubsection{Energy resources schedule results (first step)}

The first step of the application model consists on energy resource management of the distribution network to perform scheduling for each resource and determine nodal prices in each node of the network. The energy schedule and nodal prices are the basis for the application tool. Thus, Fig. 3 presents the day-ahead scheduling, regarding the first step of the proposed methodology for 24 periods during the day. One can see in this figure the high impact of the DG throughout the day, ensuring around 35\% of the energy requirements. The external suppliers are responsible for supplying around $61 \%$, and the other resources ensure the remaining $4 \%$. In fact, the ESS and V2G discharge, and mainly the DR programs only are used in very specific situations due to the higher use costs. In the present simulation scenario, a specific constraint in the external supplier's energy availability is imposed in periods 20-22 to force the use of these resources. This constraint allows the simulation of the proposed methodology considering different schedule scenarios, namely with high storage and V2G charge, with high storage and V2G discharge, with DR and considering only the loads and generators. Period 21 is the one with higher DER contribution.
The second output of the first step of the proposed methodology comprises the LMPs values in each bus. In fact, the LMPs are also different in each scheduling period resulting from different consumption/generation conditions. Due to the big diversity of the resources used, period 21 was selected for a more detailed evaluation. In Fig. 4 it is possible to see the LMP in each bus, as well as the obtained resources scheduled in each bus. Bus 0 is not considered in Fig. 4 as it is the upstream connection bus, in which the external suppliers are allocated. The external suppliers are the main suppliers of the network, and contribute with about $2229 \mathrm{~kW}$, and a LMP of $0.21 \mathrm{~m} . \mathrm{u}$./ $/ \mathrm{kWh}$. By analysing the LMPs values it is possible to verify some steps in the LMPs curve resulting from the network topology. The difference in the LMPs results from losses in a more detailed evaluation it is also possible to see that the high generation in bus 26 provided by a CHP unit results in counterflow in the branch between buses 26 and 25 . This phenomenon is reflected in the slope inversion of the LMP curve in bus 26 .

\subsubsection{Tracing algorithms results (second step)}

The second step of application model relates to the evaluation of the impact of each type of recourse in the distribution network based on two different techniques for determination of network usage. The impact that each type of resource has on a given network branch for Kirschen's and Bialek's approaches are presented in Fig. 5, which corresponds to the second stage of the methodology. Fig. 5 depicts the impact of each type of resource in each network branch. The range of the gradient color is given between the white (without impact in the branch) and black (high impact in the branch). The maximum value is of $50 \%$ due to the adopted share values presented in Table 2. Additionally, Fig. 5 is split in four areas according the network topology, so it is easier to understand and evaluate. Through Fig. 5 one can identify that the load has a high network usage, which is expected due to the fact that in each node of the distribution system there are consumption resources. The Bialek's approach results in a larger impact of the load use in the network instead of the generation. On the other hand, the Kirschen's approach shows a larger distribution of the network usage by all the energy resources. It is also noteworthy that storage units and $\mathrm{V} 2 \mathrm{G}$ resources have a greater impact on several branches of the network. This results are due to the Kirschen's approach be more simplified and less precise comparing to Bialek's approach. Thus, Kirschen's approach results in a wider distribution of the network usage, when the network tends to be meshed or if there are counterflows in the network. This aspect is more relevant in the branches between the buses 25-32.

In order to obtain a more detailed view of the proposed methodology, the resources impact in branch 11-12 are presented. This branch was selected based on the good participation of the 


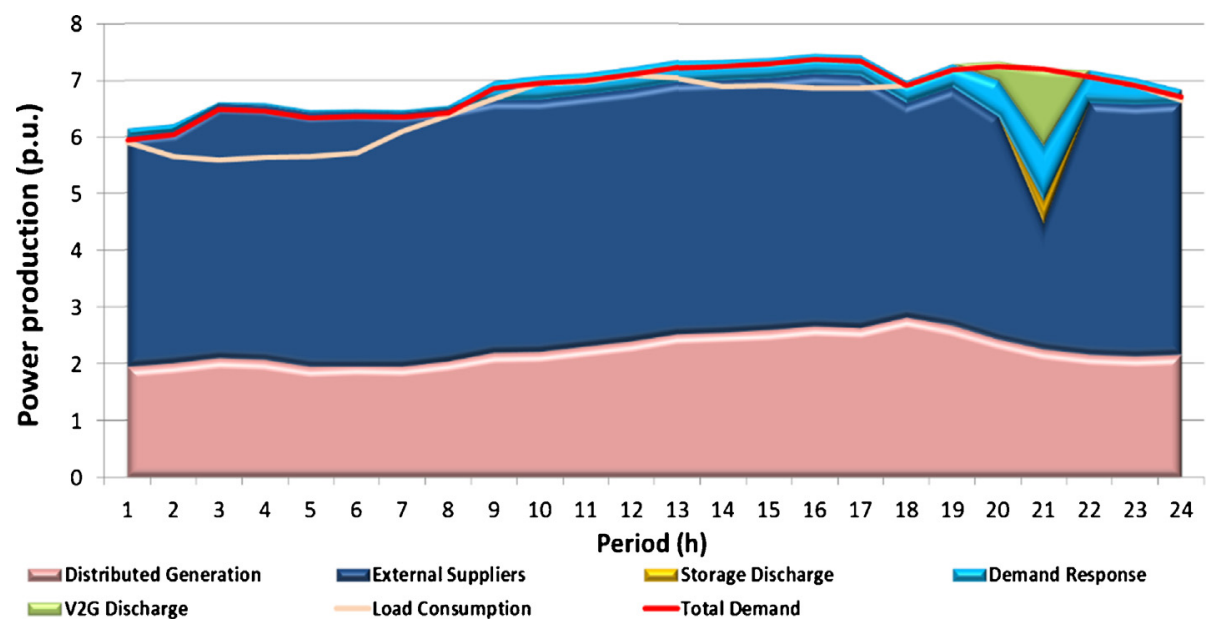

Fig. 3. Energy resources schedule in the distribution system.

different types of DER and because this branch has lower transmission capacity when compared to the remaining branches of the distribution system. Fig. 6 illustrates the share of different DER units in the power flow of branch 11-12. Moreover, it is possible to see the total costs (fixed, network use and system losses costs) that each resource has on the branch.

For the Bialek's approach, the contribution of the DG and of the external supplier in the power flow of branch 11-12 is of about $55.4 \%$, while the storage units and V2G have an impact of about $11.3 \%$ and $21.4 \%$, respectively. The remaining part (11.9\%) corresponds to the impact of loads in the branch. On the other hand, the DER participation by the Kirschen's approach is significantly different. Thus, the DG contribution in the branch $11-12$ is of about $38 \%$, while the storage and V2G resources reaches $14.5 \%$ and $26 \%$, respectively. The demand influence in the branch reaches to $21.5 \%$. The DG with greater contribution in this branch corresponds to two biomass generation units that are connected closer to branch 1112. The resource labeled as "Other DGs" corresponds to the impact of other DG units in this same branch. This portion is obtained based on the sum of all DG units with less impact in the branch. It is also possible to verify that the allocation costs follow the trend of the impact that each resource has in the network.

In general, the Bialek's approach indicates a greater share of the DER in the branch power flow than the Kirschen's approach. Regarding the V2G resources, the Kirschen's approach tends to spread the impact on the power flow through several units of V2G resources. This results in a uniform distribution of V2G by this approach.

\subsubsection{Cost allocation results (third step)}

The third step of application model is related to costs to be allocated to each resource ensuring the system economic sustainability. Each resource is charged for fixed, network use and losses costs. The results regarding the fixed, network use and systems losses costs are presented in Table 3 . The results combine all the three different philosophies of allocation costs: (i) the marginal philosophy applied to define the network use and loss cost in each branch, (ii) tracing algorithms used to determine the share of each resource in the network use, and (iii) the MW-mile used to allocate the costs for each resource taking into account the previous methods. Thus, the results comprising the combination of the three different philosophies are presented. Furthermore, a comparison of the costs considering the Bialek's and Kirschen's tracing approaches is provided for a better understanding of the proposed model. The results are divided by each type of distributed energy resource, considering the loads and external suppliers. In a generalized way, the Kirschen's approach assigns greater costs to generation resources, when compared with the Bialek's approach. On the other hand, the opposite is verified for the consumption resources. Thus, the consumption resources have a major impact on the Bialek's

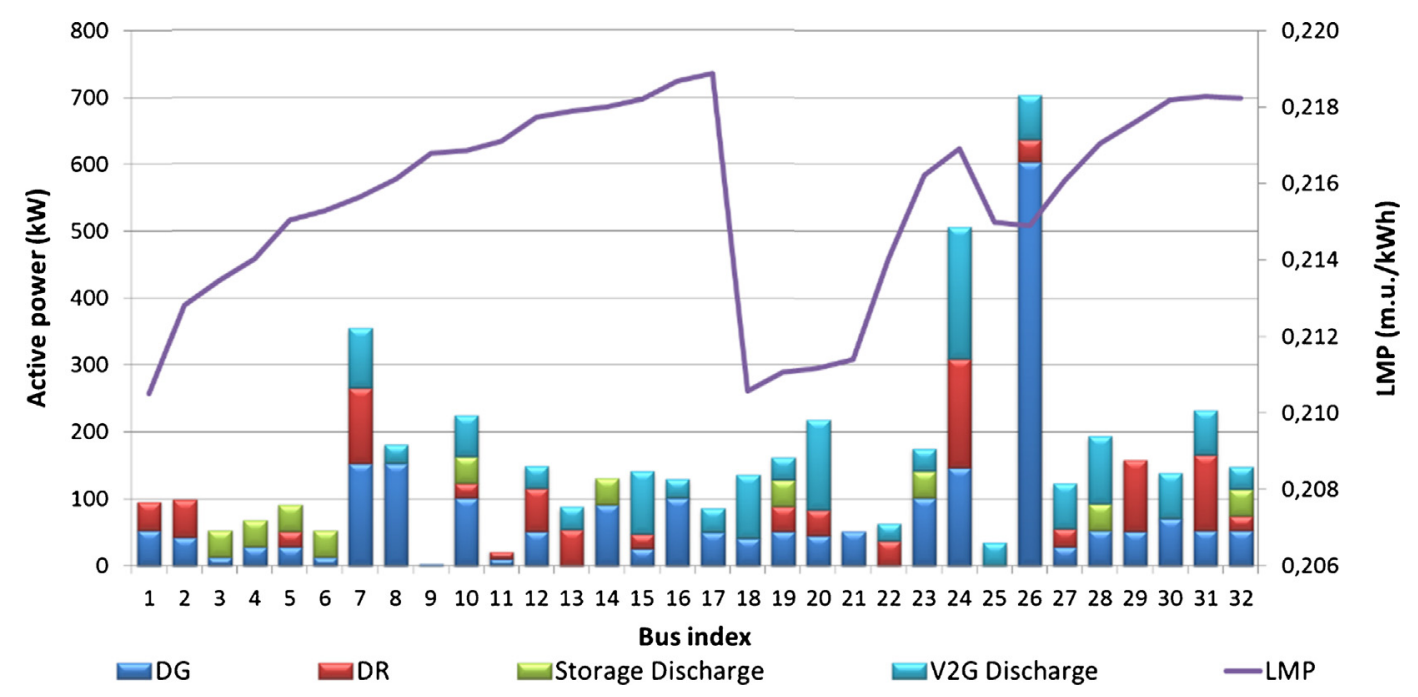

Fig. 4. Distributed energy resources dispatch and LMP by bus in period 21. 


\section{a) Kirschen's Approach}

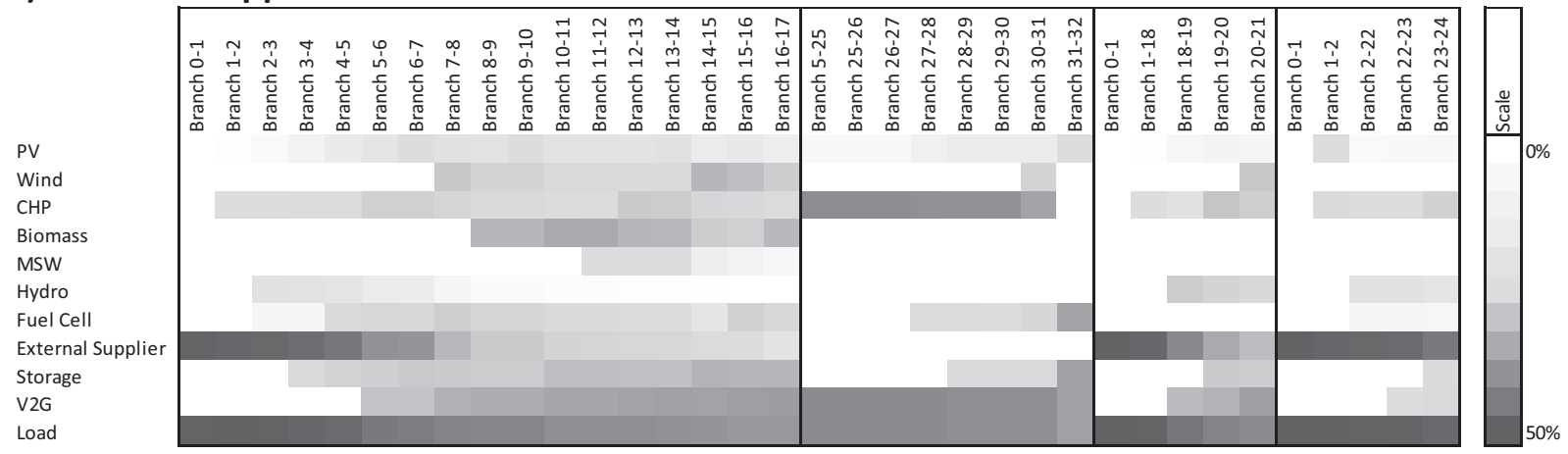

\section{b) Bialek's Approach}

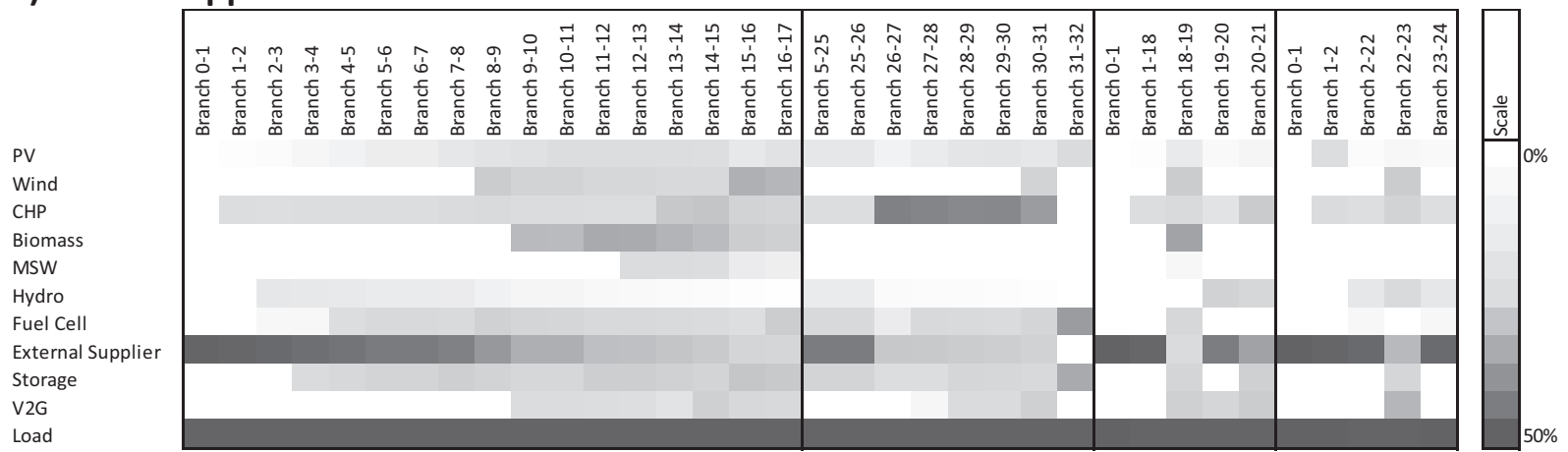

Fig. 5. Total DER impact in each branch in hour 21 considering Kirschen's and Bialek's approaches.

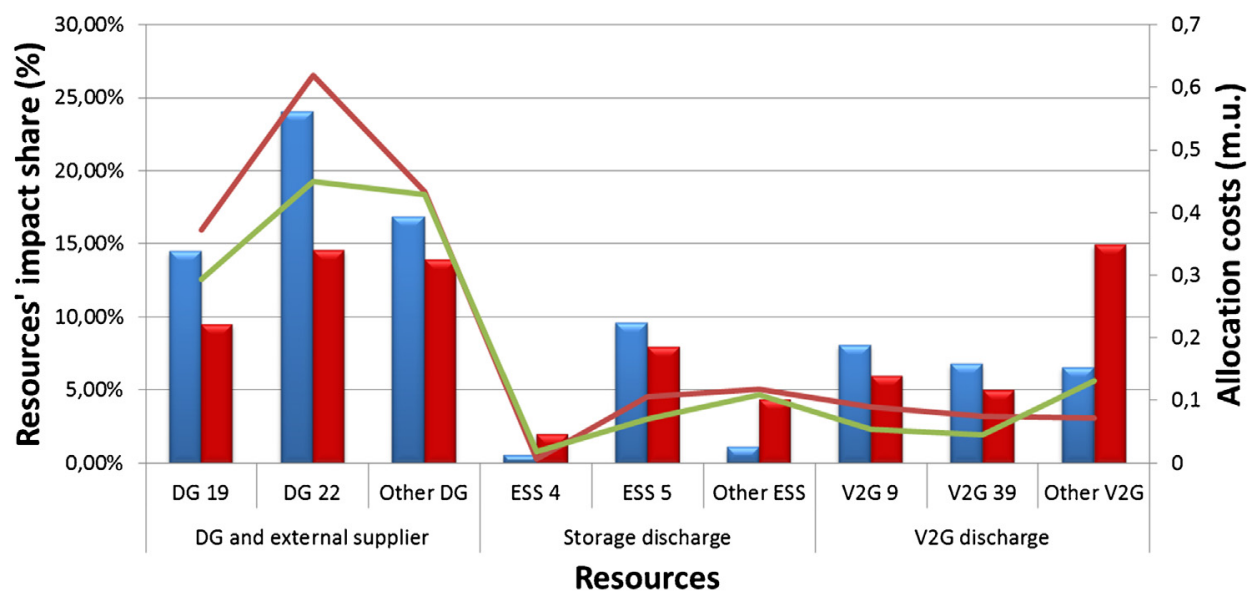

$\square$ Generation share - Bialek $\square$ Generation share - Kirschen $\longrightarrow$ Total costs - Bialek $\longrightarrow$ Total costs - Kirschen

Fig. 6. Contribution of generation resources for branch 11-12 use in period 21.

methodology than in Kirschen's. On average, the Bialek's approach allocates $70.1 \%$ of the system costs to the consumption resources, while the Kirschen's approach reaches 54.3\%. These differences result from the intrinsic characteristics and considerations of each approach, which are compounded with the penalty cost of the system's inefficiency for the consumption resources. This means that the Bialek's approach is more accurate to determine the impact of each resource in the network, yet it leads to an efficiency penalization factor in the cost allocation.

In both approaches, consumers and external supplier are the main users of the network, so they have the largest share of the total costs $-70.1 \%$ and $12.2 \%$ for Bialek's, and $54.3 \%$ and $14.5 \%$ for Kirschen's, respectively. The V2G resources take $2.78 \%$ and $7.76 \%$ of the total costs for Bialek's and Kirschen's approaches. This indicates a considerable share of these resources in the network costs, especially in Kirschen's approach.

Fig. 7 illustrates the total allocation costs for each kind of resource in each network branch considering the Bialek's and Kirschen's approaches for hour 21. The external supplier is one of the bigger providers of the network. This influence is more importance in branches closer to the upstream interconnection bus. On the other hand, the DER has major influence in the more distant branches.

Fig. 7(a) depicts the costs allocated to the energy resources for each branch of the distribution system, considering the Kirschen's tracing method. On average, the contribution of V2G resources in the branches of the distribution system is of about $17 \%$. The highest concentration of costs for V2G occurs in branch 26-27, while 
Table 3

Distribution costs allocated to DER and load.

\begin{tabular}{|c|c|c|c|c|c|c|c|c|}
\hline \multirow[t]{2}{*}{ Resources } & \multicolumn{4}{|l|}{$\begin{array}{l}\text { Bialek's } \\
\text { approach }\end{array}$} & \multicolumn{4}{|l|}{$\begin{array}{l}\text { Kirschen's } \\
\text { approach }\end{array}$} \\
\hline & $\begin{array}{l}\text { Fixed cost } \\
\text { (m.u./h) }\end{array}$ & $\begin{array}{l}\text { Power flow } \\
\text { cost (m.u./h) }\end{array}$ & $\begin{array}{l}\text { Loss cost } \\
\text { (m.u./h) }\end{array}$ & $\begin{array}{l}\text { Total costs } \\
\text { (m.u./h) }\end{array}$ & $\begin{array}{l}\text { Fixed cost } \\
\text { (m.u./h) }\end{array}$ & $\begin{array}{l}\text { Power flow } \\
\text { cost (m.u./h) }\end{array}$ & $\begin{array}{l}\text { Loss cost } \\
\text { (m.u./h) }\end{array}$ & $\begin{array}{l}\text { Total costs } \\
\text { (m.u./h) }\end{array}$ \\
\hline Photovoltaic & 0.3236 & 0.0002 & 0.0197 & 0.3435 & 0.4844 & 0.0003 & 0.0281 & 0.5128 \\
\hline Wind & 1.6908 & 0.0008 & 0.1176 & 1.8092 & 2.6839 & 0.0014 & 0.1866 & 2.8719 \\
\hline CHP & 6.8843 & 0.0055 & 0.3546 & 7.2445 & 9.5124 & 0.0072 & 0.5095 & 10.0291 \\
\hline Biomass & 3.0130 & 0.0016 & 0.2099 & 3.2246 & 4.5083 & 0.0023 & 0.3143 & 4.8249 \\
\hline WTE & 0.1020 & 0.0001 & 0.0071 & 0.1091 & 0.1364 & 0.0001 & 0.0095 & 0.1460 \\
\hline Small hydro & 0.3584 & 0.0003 & 0.0244 & 0.3831 & 0.5761 & 0.0004 & 0.0391 & 0.6156 \\
\hline Fuel cell & 1.4779 & 0.0008 & 0.0727 & 1.5514 & 2.5776 & 0.0011 & 0.1075 & 2.6862 \\
\hline External supplier & 12.2229 & 0.0168 & 0.8363 & 13.0760 & 14.5209 & 0.0182 & 0.9941 & 15.5332 \\
\hline Storage discharge & 1.0258 & 0.0005 & 0.0608 & 1.0871 & 2.8940 & 0.0013 & 0.1685 & 3.0639 \\
\hline Storage charge & 0.0000 & 0.0000 & 0.0000 & 0.0000 & 0.0000 & 0.0000 & 0.0000 & 0.0000 \\
\hline V2G discharge & 2.7801 & 0.0015 & 0.1720 & 2.9536 & 7.7563 & 0.0049 & 0.4646 & 8.2258 \\
\hline V2G charge & 0.0000 & 0.0000 & 0.0000 & 0.0000 & 0.0000 & 0.0000 & 0.0000 & 0.0000 \\
\hline Load & 70.1213 & 0.3826 & 5.0270 & 75.5308 & 54.3497 & 0.3736 & 4.0801 & 58.8034 \\
\hline Total & 100.0000 & 0.4107 & 6.9021 & 107.3129 & 100.0000 & 0.4108 & 6.9019 & 107.3128 \\
\hline
\end{tabular}

a) Kirschen's Approach

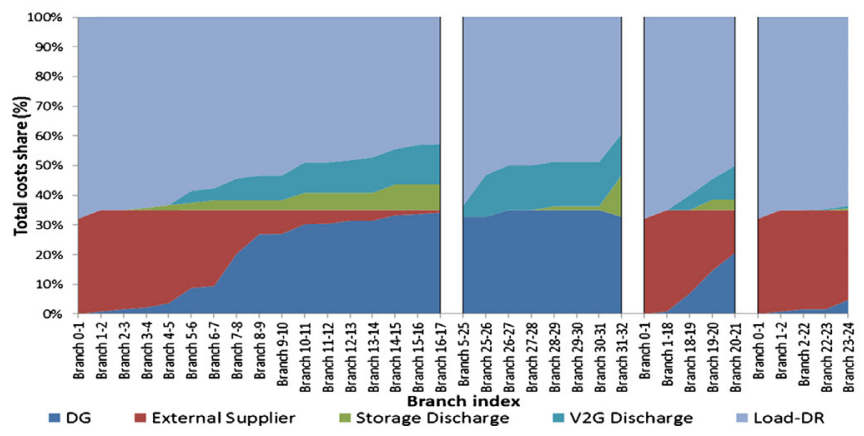

b) Bialek's Approach

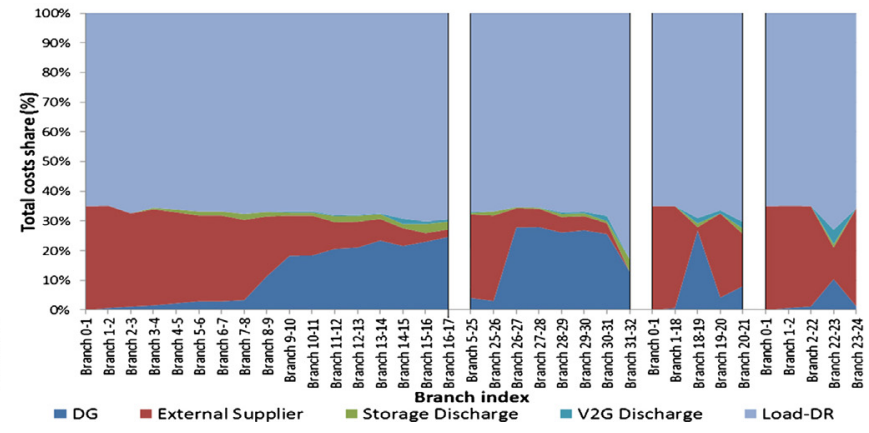

Fig. 7. Total costs in each branch by resource considering Kirschen's and Bialek's approaches in period 21.

for storage units it occurs in branch 31-32, and branch 27-28 for DG resources. Regarding the Bialek's approach, Fig. 7(b), on average, DG, storage and V2G have $46.1 \%, 3.4 \%$ and $9.3 \%$ of the total costs in the network related to generation resources, and the peak contribution is in branch 26-27, 31-32 and 20-21, respectively.

The results presented by Kirschen's and Bialek's approaches have some similarities. By comparing Fig. 7(a) and (b), it is possible to verify that the costs allocated to external suppliers in the branches closer to the upstream connection are similar. Furthermore, it seems that the DER allocation costs are higher in Fig. 7(a), which results from a lower cost allocation to the loads.

\section{Conclusions and discussion}

The main motivation behind this work is to argue for the proposal of adaption and or develop new methodologies to cost allocate and critically analyze DER in future distribution systems. In this work is proposed a methodology able to consider all types of DER in the scope of the distribution networks. The model is a hybrid methodology that uses an economic dispatch problem, Kirschen's and Bialek's tracing approaches and a variant of the MW-mile method to allocate costs to all energy resources connected to the distribution network. The model is able to determine the contribution that each resource has in each branch of the network through Kirschen's and Bialek's tracing methods, as well as to allocate different types of costs according to the LMP of each bus and based on a variant of the MW-mile method. In this way, the model provides a comparison between Kirschen's and Bialek's approaches in order to highlight the importance and impact of different approaches to the problem of tariffs allocation definition in distribution systems. In addition, it is noteworthy that Bialek's technique is more accurate in determining distribution factors than Kirschen's technique. Although both approaches are based on proportional sharing concept, Kirschen's technique use a simplified way to determine distribution factors, which is not so efficient as Bialek's technique for meshed networks and or with network counterflows.

Afterwards, the model proposes the use of three different types of costs, namely the fixed, network use and losses costs, to tariff resources and ensure network sustainability. Moreover, the model considers different tariffs approaches for each type of resource (namely, DG, DR, ESS, V2G and loads resources) taking into account their intrinsic characteristics. The two applied tracing techniques (Kirschen's and Bialek's) have different considerations for allocation costs. The contribution of each energy resource is different, resulting in different allocation costs. However, the introduction, conception and design of inefficiency penalty strategy cover the limitations of both methodologies. In this way, the overall results cover the total cost of the system operator for the network usage. Inefficiency strategy schemes for improvement of DER participation and incentive in the energy scheduled may lead to a new challenge in the way that allocation costs are spread through all types of resources. In addition, the model has the ability to adapt to different network conditions. Different network configuration and resources management leads to different results, since the model considers the integration of power flow and locational marginal costs allowing the evaluation of the network's use and to determine the allocation costs. Indeed, the proposed methodology only considers the resources that are scheduled in the first step and then fairly allocate the costs according to the network use by each 
resource. Thus, the methodology adapts itself to the network conditions in each period to fairly allocate the costs.

The use of the proposed methodology allows reaching a number of practical conclusions. The most important ones relate to (i) the adaption of tracing algorithms (usually used on transmission systems) to distribution systems with future characteristics, (ii) the noteworthy improvement of use a variant of MW-mile to cost allocate different types of resources, (iii) the impact of different types of costs in distribution system operator revenue, (iv) increasing the efficiency of the model based on a penalty scheme ensuring system operator sustainability, ( $v$ ) fairer distribution of allocation costs for all types of resources, including emerging resources, and (vi) easy adaption of the model to different network conditions.

The results support our expectations such that the model is quite diverse and able to cover several allocation costs methodologies to different types of resources. Moreover, the model effectively solves the cost allocation problem and it is illustrated by the case study considering different DER scheduling contexts.

These several conclusions are of particular relevance for several entities in electrical power systems, such as distribution operators to fair allocate all costs related to network usage, and to resources and retailers entities to simulate and evaluate its costs in network usage.

Future developments will focus on the possibility of use optimal power flow methods to deal with the unbalance on distribution networks. Finally, it will be of particular interest to analyze real-large networks with high penetration of distributed energy resources.

\section{Acknowledgments}

This work is supported by FEDER Funds through COMPETE program and by National Funds through FCT under the projects FCOMP-01-0124-FEDER: PEst-OE/EEI/UI0760/2014, and by the GID-MicroRede, project no. 34086, co-funded by COMPETE under FEDER via QREN Programme. The present work is also developed under the EUREKA - ITEA2 Project SEAS with project number 12004. Tiago Soares is partly supported by the Technical University of Denmark through a Ph.D. grant, as well as by Danish Council for strategic Research through the " 5 s - Future Electricity Markets" project (no. 12-132636/DSF).

\section{Appendix A.}

$$
\begin{aligned}
& \sum_{d g=1}^{N_{D G}^{i}}\left(P_{D G(d g, t)}^{i}-P_{G C P(d g, t)}^{i}\right)+\sum_{s p=1}^{N_{S P}^{i}} P_{S P(s p, t)}^{i} \\
& \quad+\sum_{v 2 g=1}^{N_{V 2 G}^{i}}\left(P_{D c h(v 2 g, t)}^{i}-P_{C h(v 2 g, t)}^{i}\right)+\sum_{s t=1}^{N_{S T}^{i}}\left(P_{D c h(s t, t)}^{i}-P_{C h(s t, t)}^{i}\right) \\
& -\sum_{l=1}^{N_{L}^{i}}\left(P_{L o a d(l, t)}^{i}-P_{N S D(l, t)}^{i}-P_{D R \_A(l, t)}^{i}-P_{D R \_B(l, t)}^{i}\right) \\
& =G_{i i} \times V_{i(t)}^{2}+V_{i(t)} \times \sum_{j \in L^{i}} V_{j(t)} \times\left(G_{i j} \cos \theta_{i j(t)}+B_{i j} \sin \theta_{i j(t)}\right) \\
& \sum_{D G}^{i} Q_{D G(d g, t)}^{i}+\sum_{s p=1}^{N_{S P}^{i}} Q_{S P(s p, t)}^{i}-\sum_{l=1}^{N_{L}^{i}}\left(Q_{\operatorname{Load}(l, t)}^{i}-Q_{N S D(l, t)}^{i}\right) \\
& \quad=V_{i(t)} \times \sum_{j \in L^{i}} V_{j(t)} \times\left(G_{i j} \sin \theta_{i j(t)}-B_{i j} \cos \theta_{i j(t)}\right)-B_{i i} \times V_{i(t)}^{2}
\end{aligned}
$$

$\forall t \in\{1, \ldots, T\} ; \forall i \in\left\{1, \ldots, N_{B}\right\} ; \theta_{i j(t)}=\theta_{i(t)}-\theta_{j(t)}$

$$
\begin{aligned}
& V_{\text {Min }}^{i} \leq V_{i(t)} \leq V_{\text {Max }}^{i} \\
& \theta_{\text {Min }}^{i} \leq \theta_{i(t)} \leq \theta_{\text {Max }}^{i} \\
& \left|\overline{U_{i(t)}^{-}} \times\left[\overline{y_{i j}} \times\left(\overline{U_{i(t)}^{-}}-\overline{U_{j(t)}^{-}}\right)+\bar{y}_{\overline{s h} \_i}^{-} \times \overline{U_{i(t)}^{-}}\right]^{*}\right| \leq S_{\text {Branch }(i, j)}^{\operatorname{Max}} \\
& \left|\overline{U_{j(t)}^{-}} \times\left[\overline{y_{i j}} \times\left(\overline{U_{j(t)}^{-}}-\overline{U_{i(t)}^{-}}\right)+\overline{y_{s h} j} \times \overline{U_{j(t)}^{-}}\right]^{*}\right| \leq S_{\operatorname{Branch}(i, j)}^{\operatorname{Max}} \\
& \forall t \in\{1, \ldots, T\} ; \forall i, j \in\left\{1, \ldots, N_{B}\right\} ; i \neq j ; \forall k \in\left\{1, \ldots, N_{K}\right\} \\
& 0 \leq P_{S P(s p, t)} \leq P_{\operatorname{Max}(s p, t)} \\
& 0 \leq Q_{S P(s p, t)} \leq Q_{\operatorname{Max}(s p, t)} \\
& P_{M i n(d g, t)} \times Y_{D G(d g, t)} \leq P_{D G(d g, t)} \leq P_{\operatorname{Max}(d g, t)} \times Y_{D G(d g, t)} \\
& P_{D G(d g, t)}+P_{G C P(d g, t)} \leq P_{M a x(d g, t)} \times Y_{D G(d g, t)} \\
& Q_{M i n(d g, t)} \times Y_{D G(d g, t)} \leq Q_{D G(d g, t)} \leq Q_{\operatorname{Max}(d g, t)} \times Y_{D G(d g, t)} \\
& \forall t \in\{1, \ldots, T\} ; \forall d g \in\left\{1, \ldots, N_{d g}\right\}
\end{aligned}
$$

$P_{D R \_A(l, t)} \leq P_{D R \_A ; \operatorname{Max}(l, t)}$

$P_{D R_{-} B(l, t)}=P_{D R_{-} B ; \operatorname{Max}(l, t)} \times Y_{D R_{-} B(l, t)}$

$$
\begin{aligned}
& E_{\text {Stored }(v 2 g, t)}=E_{S t o r e d(v 2 g, t-1)}-E_{\text {Trip }(v 2 g, t)}+\eta_{c(v 2 g)} \times P_{C h(v 2 g, t)} \\
& -\frac{1}{\eta_{d(v 2 g)}} \times P_{D c h(v 2 g, t)} \quad \forall t \in\{1, \ldots, T\} ; \forall v 2 g \in\left\{1, \ldots, N_{V 2 G}\right\} ; \\
& \Delta t=1 ; t=1 \rightarrow E_{\text {Stored }(v 2 g, t-1)}=E_{\text {Initial }(v 2 g)}
\end{aligned}
$$

$E_{S t o r e d}(v 2 g, t) \geq E_{\text {BatMin }(v 2 g, t)}$

$E_{\text {Stored }(v 2 g, t)} \leq E_{\text {BatMax }(v 2 g, t)}$

$P_{C h(v 2 g, t)} \leq P_{\operatorname{Max}(v 2 g, t)} \times Y_{C h(v 2 g, t)}$

$P_{D c h(v 2 g, t)} \leq P_{\operatorname{Max}(v 2 g, t)} \times Y_{\operatorname{Dch}(v 2 g, t)}$

$Y_{C h(v 2 g, t)}+Y_{D c h(v 2 g, t)} \leq 1 ; \quad Y_{C h(v 2 g, t)} \quad$ and $\quad Y_{D c h(v 2 g, t)} \in\{0,1\}$

$E_{\text {Stored }(s t, t)}=E_{S t o r e d(s t, t-1)}+\eta_{c(s t)} \times P_{C h(s t, t)}-\frac{1}{\eta_{d(s t)}} \times P_{D c h(s t, t)}$

$\forall t \in\{1, \ldots, T\} ; \forall s t \in\left\{1, \ldots, N_{S T}\right\} ; \Delta$

$t=1 ; \quad t=1 \rightarrow E_{\text {Stored }(s t, t-1)}=E_{\text {Initial }(s t)}$

$E_{\text {Stored }(s t, t)} \geq E_{\text {BatMin }(s t, t)}$

$E_{\text {Stored }(s t, t)} \leq E_{\text {BatMax }(s t, t)}$

$P_{C h(s t, t)} \leq P_{\operatorname{Max}(s t, t)} \times Y_{C h(s t, t)}$

$P_{D c h(s t, t)} \leq P_{\operatorname{Max}(s t, t)} \times Y_{\operatorname{Dch}(s t, t)}$

$Y_{C h(s t, t)}+Y_{D c h(s t, t)} \leq 1 ; \quad Y_{C h(s t, t)} \quad$ and $\quad Y_{D c h(s t, t)} \in\{0,1\}$

\section{References}

[1] P.M. De Oliveira-De Jesus, M.T. Ponce de Leão, J.M. Yusta-Loyo, H.M. Khodr Cost allocation in distribution networks with high penetration of distributed renewable generation - a comparative study, in: International Conference on Renewable Energies and Power Quality, March, Zaragoza, Spain, 2005.

[2] A.A. Abou El Ela, R.A. El-Sehiemy, Transmission usage cost allocation schemes, Electr. Power Syst. Res. 79 (6) (2009) 926-936.

[3] A. Bakirtzis, P. Biskas, A. Maissis, A. Coronides, J. Kabouris, M. Efstathiou, Comparison of two methods for long-run marginal cost-based transmission use-of-system pricing, IEEE Proc. Gener. Transm. Distrib. 148 (2001) 477-481. 
[4] R.E. Rotoras, T. Lefevre, R.B. Pacudan, Marginal transmission pricing and supplemental cost allocation method: a case of Philippines, Electr. Power Syst. Res. 63 (2002).

[5] M. Murali, M.S. Kumari, M. Sydulu, A comparison of fixed cost based transmission pricing methods, Electr. Electron. Eng. 1 (2011) 33-41.

[6] P.M.D.O.D. Jesus, M.T.P. de Leao, J.M. Yusta, H.M. Khodr, A.J. Urdaneta, Uniform marginal pricing for the remuneration of distribution networks, IEEE Trans. Power Syst. 20 (2005) 1302-1310.

[7] J. Mutale, G. Strbac, D. Pudjianto, Methodology for cost reflective pricing of distribution networks with distributed generation, in: IEEE Power Engineering Society General Meeting, 2007, 2007, pp. 1-5.

[8] D. Shirmohammadi, X.V. Filho, B. Gorenstin, M.V.P. Pereira, Some fundamental, technical concepts about cost based transmission pricing, IEEE Trans. Power Syst. 11 (1996) 1002-1008.

[9] P. Van Roy, T. Van Craenenbroeck, R. Belmans, D. Van Dommelen, G. Pepermans, S. Proost, Comparison of transmission tariff methods in a free market for electricity, in: International Conference on Electric Power Engineering, 1999. PowerTech Budapest 99, 1999, p. 159.

[10] H.H. Happ, Cost of wheeling methodologies, IEEE Trans. Power Syst. 9 (1994) $147-156$.

[11] F.J. Rubio-Oderiz, I.J. Perez-Arriaga, Marginal pricing of transmission services: a comparative analysis of network cost allocation methods, IEEE Trans. Power Syst. 15 (2000) 448-454.

[12] D. Shirmohammadi, P.R. Gribik, E.T.K. Law, J.H. Malinowski, R.E. O’Donnell, Evaluation of transmission network capacity use for wheeling transactions, IEEE Trans. Power Syst. 4 (1989) 1405-1413.

[13] R.R. Kovacs, A.L. Leverett, A load flow based method for calculating embedded, incremental and marginal cost of transmission capacity, IEEE Trans. Power Syst. 9 (1994) 272-278.

[14] J.W.M. Lima, Allocation of transmission fixed charges: an overview, IEEE Trans. Power Syst. 11 (1996) 1409-1418.

[15] M. Shahidehpour, H. Yamin, Z. Li, Market overview in electric power systems, in: Market Operations in Electric Power Systems, Wiley-IEEE Press, 2002, pp. $1-20$.

[16] P. Jiuping, Y. Teklu, S. Rahman, J. Koda, Review of usage-based transmission cost allocation methods under open access, IEEE Trans. Power Syst. 15 (2000) 1218-1224

[17] F.D. Galiana, A.J. Conejo, H.A. Gil, Transmission network cost allocation based on equivalent bilateral exchanges, IEEE Trans. Power Syst 18 (2003) 1425-1431.

[18] S.M. Harvey, W.W. Hogan, S.L. Pope, Transmission Capacity Reservations and Transmission Congeestion Contracts, FERC - Federal Energy Regulatory Comission, 1997.
[19] FERC - Federal Energy Regulatory Commission, Rated System Path Methodology, 2007.

[20] A.J. Conejo, J. Contreras, D.A. Lima, A. Padilha-Feltrin, Z_bus - transmission network cost allocation, IEEE Trans. Power Syst. 22 (2007) 342-349.

[21] J. Bialek, Topological generation and load distribution factors for supplement charge allocation in transmission open access, IEEE Trans. Power Syst. 12 (1997) 1185-1193.

[22] D. Kirschen, R. Allan, G. Strbac, Contributions of individual generators to loads and flows, IEEE Trans. Power Syst. 12 (1997) 52-60.

[23] P.M. Sotkiewicz, J.M. Vignolo, Allocation of fixed costs in distribution networks with distributed generation, IEEE Trans. Power Syst. 21 (2006) 639-652.

[24] R. Bhakar, V.S. Sriram, N.P. Padhy, H.O. Gupta, Cost allocation of DG embedded distribution system by game theoretic models, in: Power \& Energy Society General Meeting, 2009. PES'09, IEEE, 2009, pp. 1-7.

[25] T. Soares, P. Faria, Z. Vale, H. Morais, Definition of distribution network tariffs considering distribution generation and demand response, in: Presented at the 2014 IEEE Transmission \& Distribution Conference \& Exposition (T\&D2014) Chicago, USA, 2014.

[26] A.J. Conejo, E. Castillo, R. Minguez, F. Milano, Locational marginal price sensitivities, IEEE Trans. Power Syst. 20 (2005) 2026-2033.

[27] M.E. Hajiabadi, H.R. Mashhadi, LMP decomposition: a novel approach for structural market power monitoring, Electr. Power Syst. Res. 99 (6) (2013) 30-37.

[28] Á. Lorca, J. Prina, Power portfolio optimization considering locational electricity prices and risk management, Electr. Power Syst. Res. 109 (4) (2014) 80-89.

[29] M. Silva, H. Morais, T. Sousa, Z. Vale, Energy resources management in three distinct time horizons considering a large variation in wind power, in: Proceedings of EWEA Annual Event 2013, Vienna, Austria, 2013.

[30] J. Grainger, W. Stevenson, Power Systems Analysis, Mcgraw Hill, 1994.

[31] M.E. Baran, F.F. Wu, Network reconfiguration in distribution systems for loss reduction and load balancing, IEEE Trans. Power Deliv. 4 (1989) 1401-1407.

[32] M. Silva, H. Morais, Z. Vale, An integrated approach for distributed energy resource short-term scheduling in smart grids considering realistic power system simulation, Energy Convers. Manage. 64 (12)(2012) 273-288.

[33] S.B. Peterson, J. Apt, J.F. Whitacre, Lithium-ion battery cell degradation resulting from realistic vehicle and vehicle-to-grid utilization, J. Power Sources 195 (2010) 2385-2392.

[34] S.B. Peterson, J.F. Whitacre, J. Apt, The economics of using plug-in hybrid electric vehicle battery packs for grid storage, J. Power Sources 195 (2010) 2377-2384

[35] ENTSO-E, ENTSO-E overview of transmission tariffs in Europe: synthesis, in European Network of Transmission System Operators for Electricity, 2013.

[36] ERSE, Regulamento de Acesso às Redes e às interligações, in: Entidade Reguladora Dos Serviços Energéticos, 2013 (in Portuguese). 